\title{
The Ultrastructure of Basophile Leukocytes in Human Chronic Myelogenous Leukemia
}

\author{
Akira B. Miura, Akira Shibata, Seiju Onodera, \\ Atsuo Suzuki, Shinobu Sakamoto and Chiyuki Suzuki \\ The Second Department of Intemal Medicine (Prof. T. Torikai), \\ Tohoku University School of Medicine, Sendai
}

\begin{abstract}
The basophils in human chronic myelogenous leukemia with basophilia were observed by the electron microscope after fixation with aldehyde and osmium double fixatives.

The nucleus and the eytoplasmic component were almost equal in appearance to those of the neutrophils or the eosinophils except for basophilic granules. A few mitochondria of round shape were seen. The Golgi component was found usually poorly developed but cisternal component was sometimes recognized. The granular and agranular endoplasmic reticulums were poorly developed. The fibrils were often recognized.

There were many granules in cytoplasm. Their diameters reached $1.5 \mu$ and they had limiting membranes. The contents of the granules were not the same. The granules with homogeneous contents were rather few. Most of the granules contained material looking like a fibrillar structure composed of fine particles of different sizes.

These granules had a tendency to break and the membrane often disappeared. These broken vacuoles fused with each other and the cells sometimes assumed watery appearance. This is not attributed to a mere artefact in the course of fixation, but is interpreted as the morphological expression of a certain functional state of basophils.
\end{abstract}

Though many investigations of blood cells by the electron microscopy have been performed, the ultrastructure of basophils is described by only a few authors. It is because of the paucity of basophils in circulating blood and the poor fixative effect of osmic acid on these blood cells. Recently it has become clear that the aldehyde fixative is more suitable for the fixation of basophils. ${ }^{1}$

In 1966, we reported the ultrastructure of the basophils in human leukemia by osmium fixation, ${ }^{2}$ and the basophilic granules had or did not have limiting membranes and their contents looked homogeneous or fibrillar.

Recently we experienced four cases of chronic myelogenous leukemia with basophilia (Table 1). The fixation was made by glutaraldelyde and osmium tetroxide. The purpose of this paper is to describe the ultrastructure of basophils in human leukemia fixed in double fixatives.

Received for publication, May 10, 1968. 
TABLe 1. Cases of leukemia

\begin{tabular}{c|c|c|c}
\hline Number & Age & Sex & Percentage of basophils \\
1 & 40 & Male & $13.0 \%$ \\
2 & 45 & Female & $14.0 \%$ \\
3 & 46 & Male & $29.5 \%$ \\
4 & 59 & Female & $26.5 \%$
\end{tabular}

\section{Materials and Methods}

Bone marrow blood or buffy eoat of peripheral blood from the leukemic patients was fixed in 2 per cent glutaraldehyde buffered with cacodylate solution for about one hour, washed in cacodylate buffer and refixed in $1 \%$ osmie acid for one hour. After dehydration with ethanol, the samples were embedded in Epon, sectioned on a Porter-Blum ultramicrotome and examined with a Hitachi $7 \mathrm{~S}$ or HVlo type electron microscope.

The same blocks were eut at $2 \mu$ in thickness and stained with toluidine blue for optical microscopic observation.

\section{Results}

In thick sections stained with toluidine blue, the metachromatic granules of basophils were found well preserved. On electron microscopic observation, the neutrophils, eosinophils and monocytes were all fixed in good condition.

The basophils were easily identified because of their characteristic granules which were different from those of neutrophils and eosinophils. The granules of neutrophils were smaller and those of eosinophils were easily recognized by their characteristic structure. The immature basophils were rare even in chronic leukemia.

The nucleus of basophils was not different from that of other granulocytes. The chromatin of the nucleus of immature cells was less dense than that of the mature cells. The outer and inner membrane structures of the nuclear envelope were clear, and the dissociation of the outer membrane was not common. The ribosomes attached on the outer membrane were not abundant.

Generally speaking, the organelles were scarce in mature basophils except for specific granules. Occasionally a few mitochondria of round or long-shaped form were seen. The Golgi complex was poorly developed, but in rare cases the cisternal component was seen. A few vesicular agranular endoplasmic reticulums and tubular, not dilated granular endoplasmic reticulums were recognized. A few free ribosomes were also found. The fibrils were often recognized in cytoplasm. The centrioles were not rare but we did not encounter the mitotic figure. In some basophils, there were many glycogen-like particles, but in others there were almost no particles.

There were many granules in basophils. Usually they had round form. Their size was not equal but generally reached about $1.5 \mu$ in diameter. The granules had a tendency to break and great vacuoles up to $3 \mu$ appeared sometimes.

The contents of granules showed variable features. In principle they had clear limiting membranes. Sometimes they were filled with homogeneous dense 
materials with or without sub-membraneous halo, but the granules of this type were rather few. Most granules looked to be filled with fine fibrillar structure. Under higher magnifications, it was clear that these fibrillar structures were composed of fine particles. Some of fibrillar structures showed lamellar features and undulation. In other granules, the irregular dense materials were seen.

Large vacuoles without any contents or with fragments of granular materials were also recognized. The size of these vacuolar granules was extremely large, and the vacuoles had a tendency to fuse. Often the limiting membrane was found to have disappeared.

Some of the basophils were almost filled with many large vacuoles with or without the limiting membrane. They were identified as basophils because of a few residual typical basophilic granules.

The club-shaped dense materials were often seem stretching between two fusing vacuoles or protruding out of vacuoles into cytoplasm. Some of the granules contained concentric lamellar structures. They may represent a stage in the course of autolysis.

These different types of granules were recognized in a single basophil. But the characteristic of basophils was the fibrillar appearance of the granules composed of fine particles which had a tendency to break. Autophagic vacuoles or homogeneous contents of granules were also seen in the neutrophils or the eosinophils.

\section{Discussion}

Though several investigators described the ultrastructure of basophils, the results were not always the same. This is partly because of the paucity of basophils in the blood and partly of inadequacy of fixatives. The difference of species used may also have exaggerated the diversity of observations.

The observations were made in $\operatorname{man}^{1-8}$, fowls, ${ }^{9}$ rabbit ${ }^{10}$ and guinea pig. ${ }^{11-14}$ As leukemia with basophilia is suitable for the study of basophils, some authors used such cases. We also reported the ultrastructure of basophils in chronic leukemia in $1966,{ }^{2}$ and in that report we described two types of granules, one with distinct limiting membrane and homogeneous contents and the other with indistinct limiting membrane and fibrillar contents. We thought the former type might have been transformed into the latter.

Most of the authors agreed that the nucleus and organelles except for basophilic granules were equal in character to those of neutrophils and eosinophils, but in respect of specific granules, the results were different according to the investigators.

Most of the studies were made by osmium fixation and methacrylate embedding or recently Epon embedding. Recently it became also clear that the double fixation was suitable to preserve basophilic granules. Our study was made by the method almost the same as Zucker-Franklin's double fixation method, ${ }^{1}$ and in many respects our observations were in agreement with her description. 
The types of granules we observed were more variable than those described by Zucker-Franklin. There were many types of granules in basophilic leukocytes of our cases as described above. The discrepancy may be due to the difference of materials. Zucker-Franklin used the normal blood and our materials were obtained from leukemic patients under medical treatments.

Grey and Biesele, ${ }^{4}$ Goodman et al. ${ }^{5}$ and Ito $^{7}$ also reported the ultrastructure of basophils in human leukemia. Ito asserted that basophilic granules were to be classified into three types, $\alpha, \beta$, and $\gamma$-granules. Though we agree with him in recognizing that there are several forms of granules, we think it is rather difficult to classify the granule types because they have many intermediate forms. We think that in their original form the granules are round and homogeneous, but they may be so transformed as to show many variable structures, lamellar, fibrillar or irregular. But since we scarcely found immature basophils in our cases, the problem of the development of basophilic granules awaits further studies.

In our observations, we encountered many basophils with large vacuoles with or without the limiting membrane. They may assume a misleading appearance of incomplete fixation. But perfect preservation of neighboring cells and some basophilic granules in the same cell suggests that this type of basophilic granules is not simply an artefact. Of course, these granules may have dissolved in the course of fixation, but their appearance may indicate a functional state of basophils. Perhaps the basophils may assume such an appearance after discharging a certain chemical substance, presumably histamine.

Though Winquist ${ }^{13}$ asserted that liberation of histamine did not result in any morphological changes of granules, we think it is likely that the secreting function is associated with the fragility of basophilic granules. In our case 3, the amount of histamine in the blood, plasma and urine of the patient were 10 to 50 times that of normal subjects. ${ }^{2}$ We think that the leukemic state or antileukemic treatment may have relation to the vacuole formation of basophils.

Fibrils were seen in many basophils. This structure may be recognized in any type of the cells and it is said that fibrils are parts of the cytoskeletal system, though they are most easily seen in neoplastic ${ }^{15}$ or activated cells. ${ }^{16}$ It is likely that we could easily recognize the fibrils because our materials were obtained from leukemic patients.

\section{Acknowledgment}

We wish to express our thanks to Prof. T. Torikai for his guidance.

\section{References}

1) Zucker-Franklin, D. Electron microscopic study of human basophils. Blood, 1967,

2) Shibata, A., Takase, S., Onodera, S., Miura, A.B., Suzuki, A., Sakamoto, S., Oikawa, A. \& Sato, T. Cytological studies on basophil leucocytes. Acta hremat. jap.,

3) Kautz, J. \& DeMarsh, Q.B. An electron microscope study of sectioned cells of 
peripheral blood and bone marrow. Blood, 1954, 9, 24-38.

4) Grey, C.E. \& Biesele, J.J. Thin-section electron microscopy of circulating white blood cells. Rev. d'Hémat. 1955, 10, 283-299.

5) Goodman, J.R., Reilly, E.B. \& Moore, R.E. Electron microscopy of formed elements of normal human blood. Blood, 1957, 12, 428-442.

6) Braunsteiner, H. \& Pakesch, F. Elektronenmikroskopische Untersuchungen der Granula menschlicher Leukozyten. Acta haemat., 1957, 17, 136-142.

7) Ito, T. Electron microscopic observation on myeloid leukemic cells, with special reference to the findings resembling virus infection. Acta hacemat. jap., 1958, 21, $631-642$.

8) Low, F.N. \& Freeman, J.A. Electron Microscopic Atlas of Normal and Leukemic Human Blood. Mograw-Hill Book Co., New York-Tronto-London, 1958.

9) Osako, R. An electron microseopic observation on the specific granules of eosinophil leukocytes of vertebrates. Acta homat. jap., 1959, 22, 134-150.

10) Wetzel, B.K., Horn, R.G. \& Spicer S.S. Fine structural studies on the development of heterophil, eosinophil, and basophil granulocytes in rabbits. Lab. Invest., 1967, 16, 349-382.

11) Pease, D.C. Marrow cells seen with the electron microscope after ultrathin sectioning. Rev. d'Hémat., 1955, 10, 300-313.

12) Pease, D.C. An electron microscopic study of red bone marrow. Blood, 1956, 11, $501-526$.

13) Winquist, G. Electron microscopy of the basophilic granulocyte. Ann. New York Acad. Sci., 1963, 103, 352-375.

14) Fawcett, D.W. An Atlas of Fine Structure. The Cell, Its Organelles and Inclusions. W.B. Saunders Co., Philadelphia and London, 1966, p. 201.

15) Freeman, J.A. \& Samuels, M.S. The ultrastructure of a "fibrillar formation" of leukemic human blood. Blood, 1958, 13, 725-731.

16) Parker, J.W., Wakasa, H. \& Lukes, R.J. Cytoplasmic fibrils in mixed lymphocyte cultures. Blood, 1967, 29, 608-615. 
Fig. 1. A basophil with granules of various types. $\times 9,600$.

Fig. 2. Well developed Golgi complex. $\times 16,900$.

Fig. 3. Granules composed of fine particles. They looked fibrillous. $\times 17,700$. 


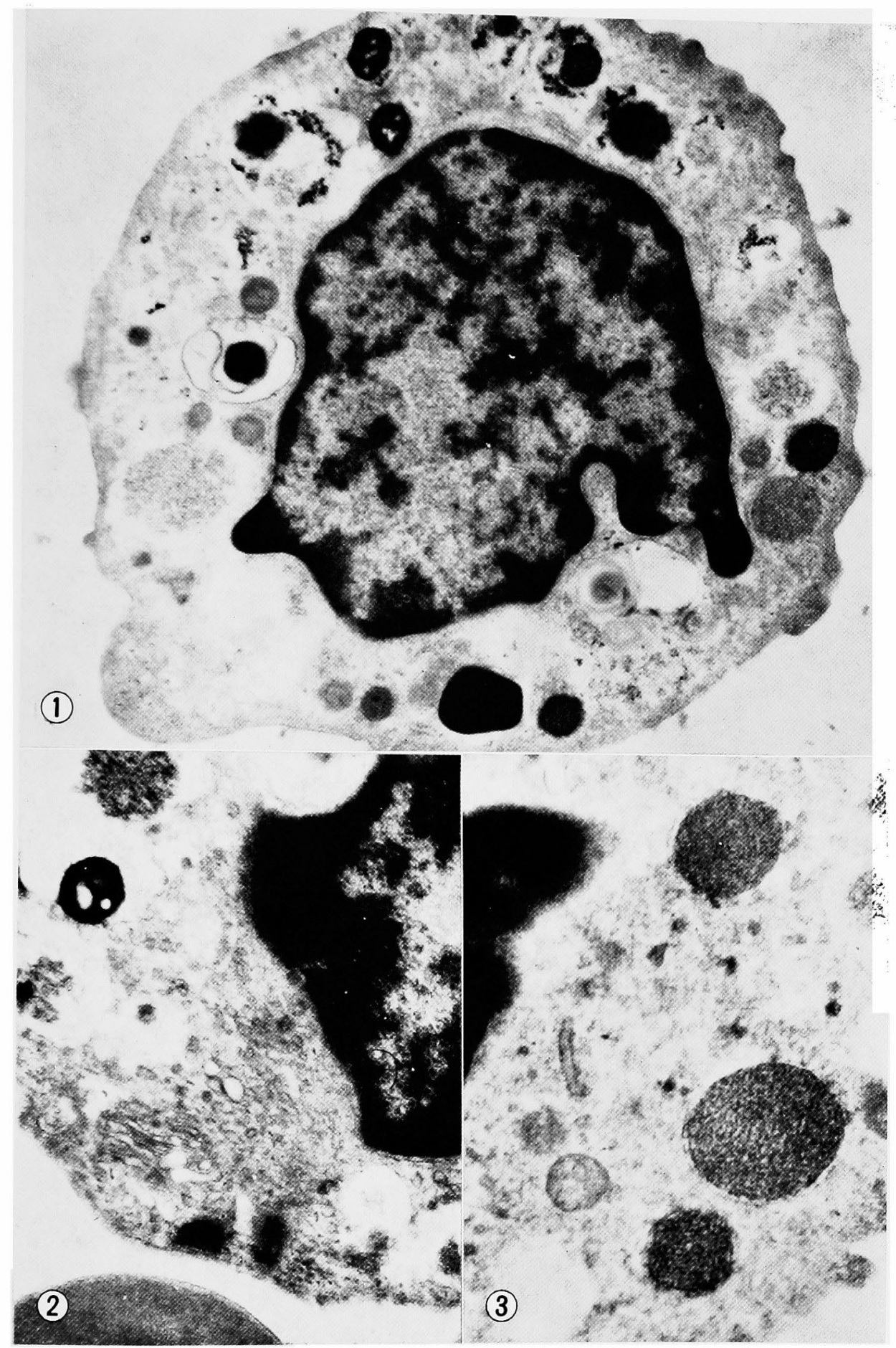


Fig. 4. Club-shaped dense materials protruding out of vacuoles. Concentric lamellar structures were also seen. $\times 9,700$.

Fig. 5. Fibrils in cytoplasm. $12,400$.

Fig. 6. A basophil with large vacuoles. $\times 11,000$. 


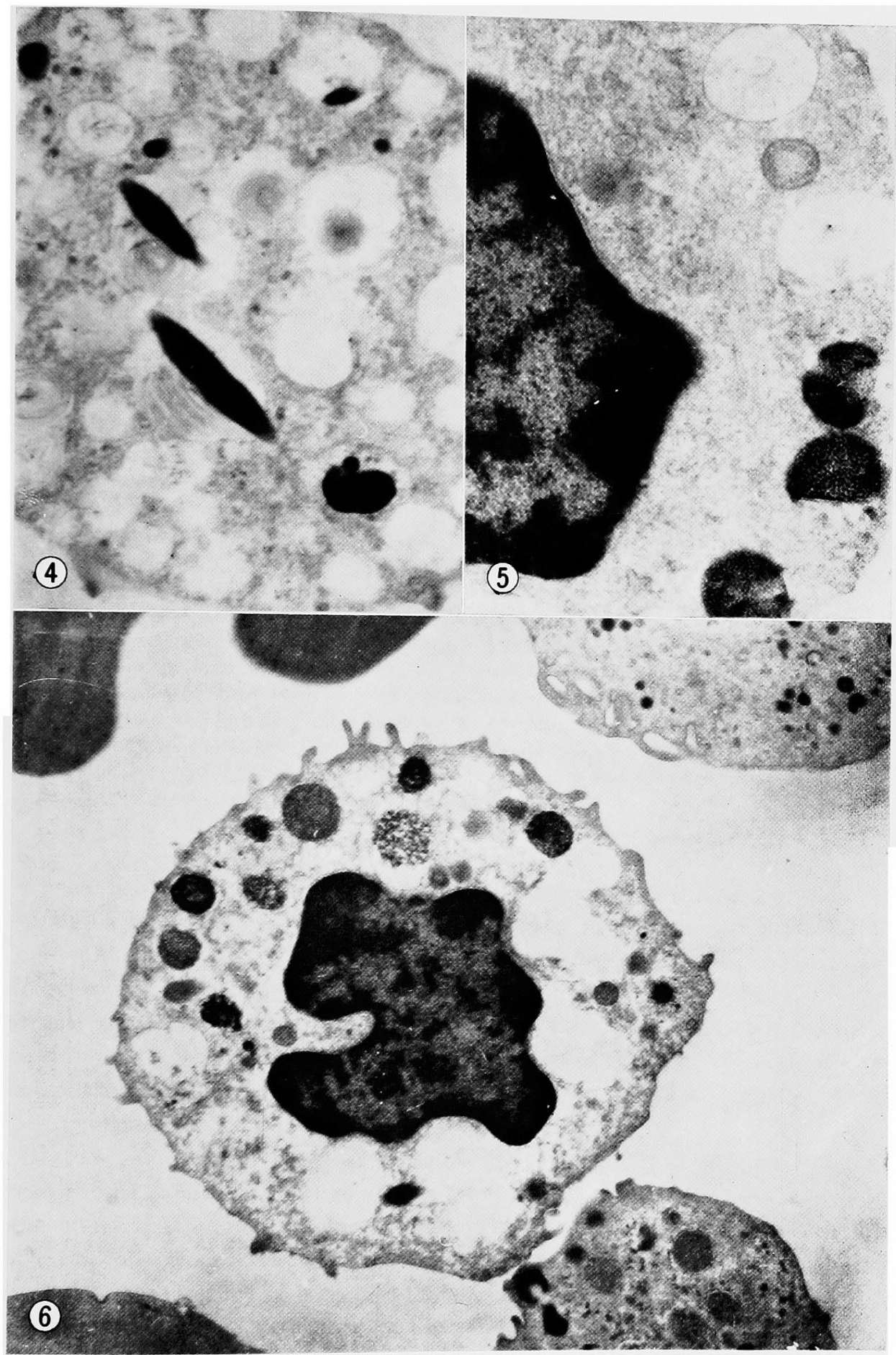

